\title{
Cause-specific mortality trends in a nationwide population-based cohort of childhood-onset type 1 diabetes in Japan during 35 years of follow-up: the DERI Mortality Study
}

\author{
Aya Morimoto $\cdot$ Yoshiko Onda $\cdot$ Rimei Nishimura • \\ Hironari Sano • Kazunori Utsunomiya • Naoko Tajima • \\ For The Diabetes Epidemiology Research International (DERI) Mortality Study Group
}

Received: 23 April 2013 /Accepted: 28 June 2013 /Published online: 1 August 2013

(C) Springer-Verlag Berlin Heidelberg 2013

\begin{abstract}
Aims/hypothesis The aim of this study was to investigate long-term, cause-specific mortality trends among patients with childhood-onset type 1 diabetes in Japan.

Methods Individuals included in the study had received a diagnosis of type 1 diabetes at age $<18$ years between 1965 and 1979. All individuals were followed up for their survival status until 1 January 2005. The causes of death were divided into end-stage renal disease (ESRD), acute diabetic complications (ADC), accident/suicide, cardiovascular disease (CVD), infections, cancers, others (non-diabetic/diabetic) and unknown. The cause-specific mortality trends were expressed according to the follow-up period and year of diagnosis.

Results A total of 1,385 patients were enrolled in the study, and the survival status of 1,324 was confirmed. Mortality rate at the 35 year follow-up (per 100,000 person-years) was
\end{abstract}

Members of the DERI study group are given in the Appendix.

Electronic supplementary material The online version of this article (doi:10.1007/s00125-013-3001-2) contains peer-reviewed but unedited supplementary material, which is available to authorised users.

A. Morimoto $(\bowtie) \cdot$ Y. Onda $\cdot$ R. Nishimura $\cdot$ H. Sano $\cdot$

K. Utsunomiya

Division of Diabetes, Metabolism and Endocrinology, Department

of Internal Medicine, Jikei University School of Medicine, 3-25-8

Nishishinbashi, Minato-ku, Tokyo 105-8461, Japan

e-mail: aya@jikei.ac.jp

R. Nishimura

Graduate School of Public Health, University of Pittsburgh,

Pittsburgh, PA, USA

N. Tajima

Department of Internal Medicine, Jikei University School of

Medicine, Tokyo, Japan
659.3, and the standardised mortality ratio (SMR) was 10.7 . The SMR at the 25 year follow-up markedly declined from 19.3 in the 1965-1969 diagnosis group to 6.6 in the 1975 1979 diagnosis group. Approximately $40 \%$ died of ADC among those with $<10$ years of follow-up. A similar proportion of individuals died of ESRD among those with 10 19 years of follow-up. The longer the duration of followup, the lower the mortality from ADC and the greater the mortality from CVD.

Conclusions/interpretation In Japanese people with childhoodonset type 1 diabetes of more than 20 years of duration, CVD was the leading cause of death, as is the case among similar white people. The longer the duration of diabetes, the more attention should be paid to preventing CVD.

Keywords Cause of death $\cdot$ Mortality $\cdot$ SMR $\cdot$ Type 1 diabetes

\section{Abbreviations}

ADC Acute diabetic complications

CVD Cardiovascular disease

DERI Diabetes Epidemiology Research International

ESRD End-stage renal disease

SMR Standardised mortality ratio

\section{Introduction}

The annual incidence of type 1 diabetes mellitus among children in Japan is 2.4 per 100,000 , which is $1 / 24$ th of the 57.6 ratio seen in Finland [1], a country with one of the highest incidences of type 1 diabetes in the world. Whether the low incidence of the disease might be related to underlying conditions that would affect the clinical course of type 
1 diabetes is unknown, as there are few population-based studies investigating the long-term prognosis. Therefore, the population-based Diabetes Epidemiology Research International (DERI) mortality study was launched in 1986 as an observational international collaborative study that brought together diabetes researchers from Finland, the USA, Israel and Japan.

In 1991, the study issued its first report demonstrating that Japanese patients with type 1 diabetes had a much worse prognosis than did similar patients in Finland, the USA and Israel, with the most frequent cause of death being end-stage renal disease (ESRD) $[2,3]$. The current study has evaluated the latest data identifying living status and cause-specific mortality trends in Japan, as of 2005.

\section{Methods}

Individuals The study comprised participants who received a diagnosis of type 1 diabetes at $<18$ years of age between 1965 and 1979; these data were retrieved from two nationwide surveys conducted on childhood-onset diabetes in 1970 and 1981, as described elsewhere [2]. Type 1 diabetes was defined as requiring initiation of insulin therapy after diagnosis. Individuals who received their diagnoses between 1965 and 1969 started follow-up on 1 January, 1970, and those who received their diagnoses between 1970 and 1979 started follow-up on 1 January, 1980. All individuals were alive on the day when the follow-up started.

This cohort comprised 1,385 patients, 23 fewer than were evaluated in the survey that determined living status as of 1995 [4], owing to a correction for misclassifications and violations of the inclusion criteria discovered during the past 10 years. Case-ascertainment of the cohort was estimated to be $75 \%$, according to the reported type 1 diabetes incidence rate $(0.8$ per 100,000 person-years $)$ during that period. The representativeness of the cohort to the target population is discussed elsewhere $[2,5]$.

Methods of follow-up A questionnaire to confirm an individual's vital status was mailed to each individual's attending physician every 5 years (Electronic supplementary material [ESM] questionnaire). All individuals were followed up until 1 January 2005. Any individual whose status was unknown was further followed up by certificates of registry or copies of his or her family register with the approval of the Ministry of Justice. Survival status was expressed in terms of mortality rate and standardised mortality ratio (SMR).

Determination of causes of death The causes of death were determined by the DERI mortality classification committee [3] based on the information from attending physicians or death certificates. The causes of death were divided into nine categories: (1) ESRD, (2) acute diabetic complications (ADC), (3) accident/suicide, (4) cardiovascular disease (CVD), (5) infections, (6) cancers, (7) other non-diabetic causes, (8) other diabetic causes, and (9) unknown.

Statistical analysis SMRs were calculated by using the annual mortality rates and the annual cause-specific mortality rates from 1970 to 2004 released by the Japanese Ministry of Health, Labour and Welfare. The 95\% CIs were calculated by using the Poisson distribution [6]. Statistical analyses were conducted by using SAS 9.3 (SAS Institute, Cary, NC, USA). A $p$ value less than 0.05 was considered to be statistically significant (two-sided test). The study was approved by the institutional review board of Jikei University School of Medicine and was carried out in accordance with the Declaration of Helsinki.

\section{Results}

Among the total of 1,385 children with type 1 diabetes ( 556 boys and 829 girls; mean [ $\pm \mathrm{SD}$ ] age at diagnosis, $8.8 \pm 4.1$ years), 1,101 were confirmed as alive as of 2005 , and 223 deaths were observed. Of the 61 individuals whose status remained unknown, their last confirmed status before 2005 was used for analysis. The mean duration of diabetes was $27.9 \pm 6.5$ years, with a mean follow-up of $24.4 \pm 6.4$ years, and a mean age at death of $30.7 \pm 8.4$ years.

All-cause mortality and SMRs The mortality rate (per 100,000 person-years) and the SMR at 35 year follow-up were 659.3 and 10.7 (95\% CI 9.3, 12.1), respectively. The mortality rate was shown to be higher among male than female participants (778.1 vs 580.5); however, the SMR was lower among men than women (9.6 vs 14.3), because of a higher mortality rate among men in the general population. The mortality at 25 year follow-up was considerably improved in the 1975-1979 diagnosis group compared with that in the 1965-1969 diagnosis group (SMR, 6.6 vs 19.3).

Cause-specific mortality and SMRs Overall, the leading causes of death were ESRD and CVD, followed by ADC (Table 1). ESRD was also the most frequent cause of death in the 1965-1969 diagnosis group, at $42 \%$ of all causes of death. However, it decreased dramatically in the 19751979 diagnosis group. In men, the leading causes of death were CVD, ADC and infections. ESRD was the fourth highest cause of death in men. In women, ESRD was the highest cause of death, followed by CVD, and ADC.

Total cause-specific SMRs at 35 year follow-up, and at 25 year follow-up for the 1965-1969 and 1975-1979 


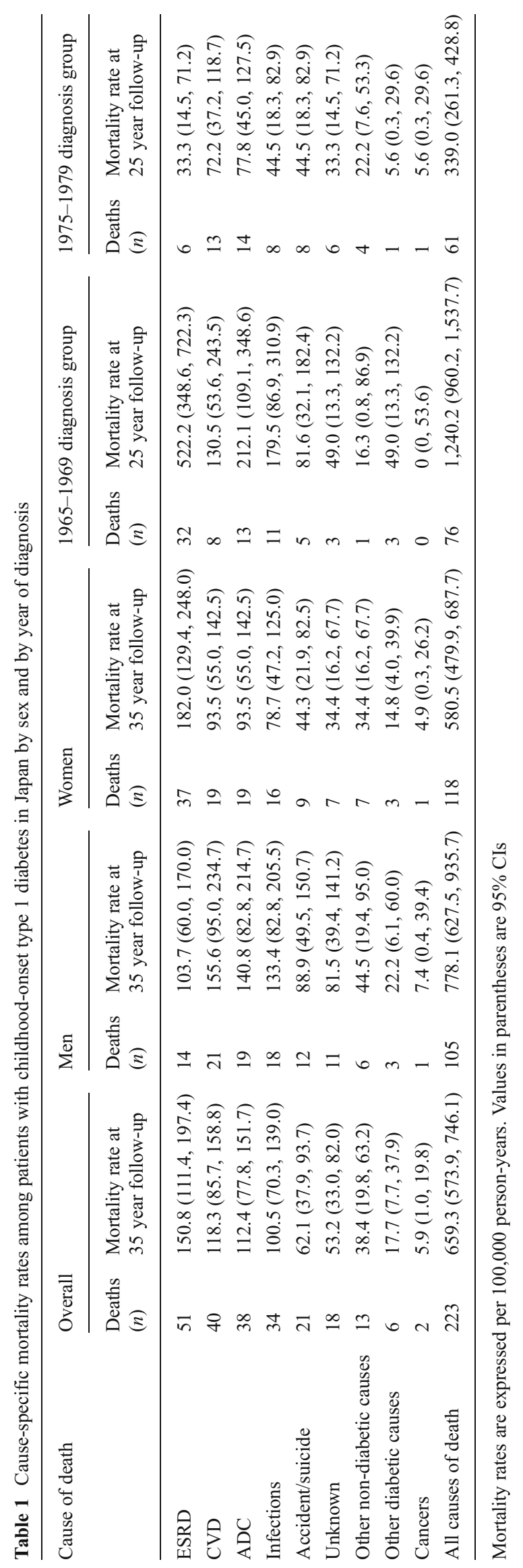


diagnosis groups, respectively, are as follows: CVD, 13.8/16.9/11.6; infections, 46.9/61.7/27.3; accident/suicide, 2.1/2.6/1.6; cancers 0.5/0.0/0.6. Details of cause-specific SMRs and their trends are available in the ESM Tables 1 and 2 .

Among fatal ADC, diabetic ketoacidosis was the most frequent $(50.0 \%)$, followed by diabetic coma unspecified $(28.9 \%)$, and hypoglycaemia $(21.1 \%)$. In the accident/suicide category, suicide was the most frequent (57.1\%). In the CVD category, cerebral haemorrhage was the most frequent $(37.5 \%)$, followed by myocardial infarction $(20.0 \%)$ and heart failure $(17.5 \%)$. Among the fatal infections, sepsis was the most frequent $(35.3 \%)$, followed by pneumonia $(29.4 \%)$. The cancers, of which two patients died, were Wilms' tumour and gastric cancer. Other nondiabetic causes of death included gastric ulcer (15.4\%) and pancreatitis (15.4\%). Among other diabetic causes, gangrene was the most frequent (50.0\%).

We found that the longer the duration of follow-up, the lower the mortality from ADC, and the greater the mortality from CVD (Fig. 1).

\section{Discussion}

In our previous report in 1991 [3], ESRD and ADC were the leading causes of death, and CVD was rare. However, the current analyses have revealed that the longer the duration of follow-up, the higher the percentage of CVD in total death, with CVD becoming the leading cause of death at the 30 35 year follow-up period. The increasing trend of CVDrelated mortality was also reported in the USA DERI cohort [7]. For the Japanese general population, the age-adjusted CVD-related mortality rate has decreased in recent years [8], and it is known that mortality due to CVD is relatively low compared with that in the white population [9]. Therefore, it is interesting to reveal this increasing trend of CVD-related mortality in the Japanese DERI cohort.

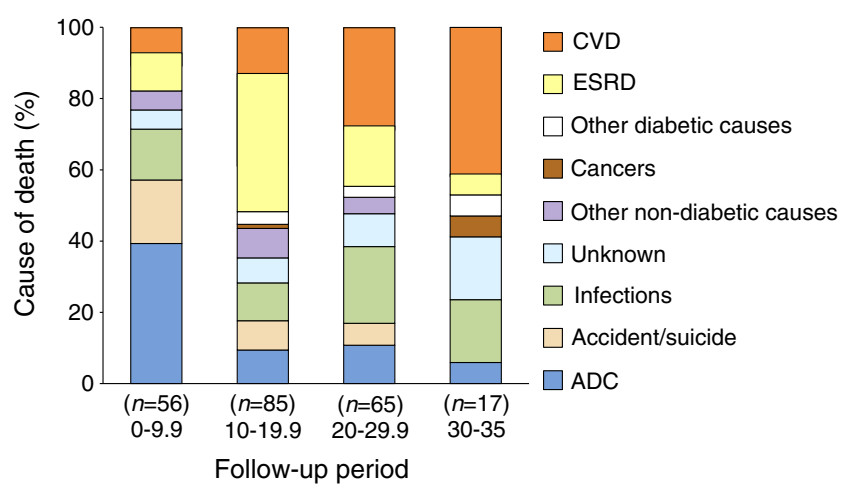

Fig. 1 The causes of death according to follow-up period in patients with childhood-onset type 1 diabetes in Japan
Our previous report in 1995 revealed that the ESRDrelated mortality risk in our cohort was 2.57 times higher than that seen in the USA DERI cohort [10]. The large difference in ESRD-related mortality between these two countries was reduced considerably after the 30 year follow-up. The ESRD-related mortality rate (per 100,000 person-years) in 2008 in the USA cohort was 88.5 in men and 129.0 in women [7], thus becoming closer between the two countries, particularly among men. It was more difficult to initiate dialysis during the late 1960s and 1970 s in Japan because of the limited number of dialysers, and the fact that many families were reluctant to pay for dialysis treatment until a medical care benefit system that covered high-cost healthcare was established in 1981. Moreover, working men were more likely than women to be given priority to receive dialysis or to be offered it at an earlier stage in their disease.

The SMR for infections was 46.9 overall. It was particularly high in the 1965-1969 diagnosis group and declined by half in the 1975-1979 diagnosis group. A marked improvement in both the management of type 1 diabetes and in infectious diseases might have ameliorated the SMR of premature death. The SMR for cancer was 0.5. Although patients with type 2 diabetes have a higher risk of cancer than has the general population, the main contributing mechanism is insulin resistance [11], which is not usually seen in type 1 diabetes in Japan [12]. A limitation of the present study is that individuals' clinical data, such as glycaemic status, hypertension or dyslipidaemia, were not considered in the analysis.

In conclusion, the mortality risk for a patient receiving a diagnosis of childhood-onset type 1 diabetes in Japan between 1965 and 1979 was 10.7-fold higher than that of the general Japanese population at the 35 year follow-up. However, the SMR at the 25 year follow-up markedly improved from 19.3 in the 1965-1969 diagnosis group to 6.6 in the 1975-1979 diagnosis group. As the duration of followup increased, ADC contributed less and CVD contributed more to mortality. As in white populations, among Japanese people the longer the duration of type 1 diabetes, the more attention should be paid to preventing CVD.

Acknowledgements The authors thank the study participants, attending physicians and investigators of the DERI mortality study group for their contributions to the study. The authors also thank the members of the Mortality Classification Committee: Trevor J. Orchard (University of Pittsburgh, Graduate School of Public Health, Pittsburgh, Pennsylvania, USA) and Jaakko Tuomilehto (Department of Public Health, University of Helsinki, Helsinki, Finland) for their assistance.

Funding This study was supported by grants from the National Institutes of Health (DK-35905); the Ministry of Health and Welfare, Japan (H10Kodomo-022); and the Ministry of Education, Culture, Sports, Science, and Technology, Japan (Wakate-B 14770186, 16790328, 22790573). 
Duality of interest The authors declare that there is no duality of interest associated with this manuscript.

Contribution statement AM collected data, edited the database, analysed data and wrote the manuscript. YO and HS collected data and edited the database. RN collected data, edited the database and reviewed/edited the manuscript. KU interpreted the data and reviewed the manuscript. NT designed and started the study, collected data, constructed the database and reviewed/edited the manuscript. All authors critically revised the manuscript and approved the final version of the manuscript.

\section{Appendix}

The DERI Study Group (Japan) includes the following members (alphabetical order by last name): Masakazu Haneda (Division of Metabolism and Biosystemic Science, Department of Medicine, Asahikawa Medical College, Asahikawa), Shohei Harada (Department of Health Policy, National Research Institute for Child Health and Development, National Center for Child Health and Development, Setagaya), Nigishi Hotta (Department of Diabetes and Endocrinology, Chubu Rosai Hospital, Nagoya), Hideaki Jinnouchi (Jinnouchi Clinic, Diabetes Care Center, Kumamoto), Tomoyuki Kawamura (Department of Pediatrics, Osaka City University Graduate School of Medicine, Osaka), Nobuyuki Kikuchi (Department of Pediatrics, Yokohama City Minato Red Cross Hospital, Yokohama), Susumu Konda (Konda Children's Clinic, Chiba), Masae Minami (Minami Masae Clinic, Fukuoka), Aya Morimoto (Division of Diabetes, Metabolism and Endocrinology, Department of Internal Medicine, Jikei University School of Medicine, Minato), Rimei Nishimura (Division of Diabetes, Metabolism and Endocrinology, Department of Internal Medicine, Jikei University School of Medicine, Minato), Yoshiko Onda (Division of Diabetes, Metabolism and Endocrinology, Department of Internal Medicine, Jikei University School of Medicine, Minato), Hironari Sano (Division of Diabetes, Metabolism and Endocrinology, Department of Internal Medicine, Jikei University School of Medicine, Minato), Naoko Tajima (Department of Internal Medicine, Jikei University School of Medicine, Minato), Akira Takeda (Tottori Prefecture Chuo Hospital, Tottori), Koji Takemoto (Department of Pediatrics, Ehime University School of
Medicine, Toon), Takayoshi Toyota (Division of Molecular Metabolism and Diabetes, Tohoku University Graduate School of Medicine, Sendai), Yasuko Uchigata (Diabetes Center, Tokyo Women's Medical University School of Medicine, Shinjyuku), and Tatsuhiko Urakami (Department of Pediatrics, Nihon University School of Medicine, Itabashi)

\section{References}

1. International Diabetes Federation (2011) IDF diabetes atlas, 5th edn. International Diabetes Federation, Brussels, Belgium

2. Diabetes Epidemiology Research International Mortality Study Group (1991) Major cross-country differences in risk of dying for people with IDDM. Diabetes Care 14:49-54

3. Diabetes Epidemiology Research International Mortality Study Group (1991) International evaluation of cause-specific mortality and IDDM. Diabetes Care 14:55-60

4. Asao K, Sarti C, Forsen T et al (2003) Long-term mortality in nationwide cohorts of childhood-onset type 1 diabetes in Japan and Finland. Diabetes Care 26:2037-2042

5. The Diabetes Epidemiology Research International (DERI) Study (1995) International analysis of insulin-dependent diabetes mellitus mortality: a preventable mortality perspective. Am J Epidemiol 142:612-618

6. Crow EL, Gardner RS (1959) Confidence intervals for the expectation of a Poisson variable. Biometrika 46:441-453

7. Secrest AM, Becker DJ, Kelsey SF, Laporte RE, Orchard TJ (2010) Cause-specific mortality trends in a large population-based cohort with long-standing childhood-onset type 1 diabetes. Diabetes 59:3216-3222

8. Statistics and Information Department, Minister's Secretariat, Ministry of Health, Labour and Welfare (2010) Vital statistics of Japan 1: 324-329

9. World Health Organization, the Global Health Observatory; Noncommunicable diseases: Mortality: Cardiovascular diseases and diabetes, deaths per 100,000. http://apps.who.int/gho/data/. Accessed 23 February 2013

10. Matsushima M, Tajima N, LaPorte RE et al (1995) Markedly increased renal disease mortality and incidence of renal replacement therapy among IDDM patients in Japan in contrast to Allegheny County, Pennsylvania, USA. Diabetologia 38:236-243

11. Johnson JA, Carstensen B, Witte D, Bowker SL, Lipscombe L, Renehan AG, Diabetes and Cancer Research Consortium (2012) Diabetes and cancer (1): evaluating the temporal relationship between type 2 diabetes and cancer incidence. Diabetologia 55:16071618

12. Arai K, Yokoyama H, Okuguchi F et al (2008) Association between body mass index and core components of metabolic syndrome in 1486 patients with type 1 diabetes mellitus in Japan (JDDM 13). Endocr J 55:1025-1032 